\title{
Admissible rules of modal logics
}

\author{
Emil Jeřábek* \\ Department of Philosophy \\ Utrecht University \\ Heidelberglaan 8 \\ 3584 CS Utrecht, The Netherlands \\ jerabek@math.cas.cz
}

\begin{abstract}
We construct explicit bases of admissible rules for a representative class of normal modal logics (including the systems $K 4, G L, S 4, G r z$, and $G L .3$ ), by extending the methods of S. Ghilardi and R. Iemhoff. We also investigate the notion of admissible multiple conclusion rules.
\end{abstract}

\section{Introduction}

The research of derivation in logical systems or formal theories usually focuses on axioms and theorems; nevertheless, the investigation of inference rules turns out to be a fruitful generalization of the questions studied in proof theory. One of the most remarkable features of rules, not present in the theoremhood area, is the distinction between derivable and admissible rules. The latter concept goes back to P. Lorenzen [19]; a schematic rule is admissible in a formal system $S$, if the set of theorems of $S$ is closed under the rule. Description of the structure of admissible rules of a given formal system may have a practical value (admissible rules form the largest collection of inference rules which can be used in derivations while preserving the set of theorems), but more significantly, it provides an important insight in the general properties of the system.

The modern development of the theory of rules admissible in non-classical logics was inspired by a question of H. Friedman [9], whether admissibility in intuitionistic logic is decidable. The problem was thoroughly studied in the 80 's and 90 's in a series of papers by V. Rybakov, later summarized in the book [20]. Rybakov's deep results on semantical characterization of admissibility have provided decision procedures for admissible rules in many modal and superintuitionistic logics (thus answering positively Friedman's question), and settled various properties of sets of admissible rules and their bases (e.g., non-existence of finite bases of admissible rules for IPC and certain modal logics).

Another direction of research stemmed recently from results of S. Ghilardi on unification in intuitionistic and modal logics $[10,11]$, which provided a different characterization of

\footnotetext{
*Partially supported by grant IAA1019401 of GA AV ČR.
} 
admissibility. R. Iemhoff [13] discovered how to use these results to construct explicit bases of admissible rules; specifically, she proved completeness of a basis for IPC earlier conjectured by D. de Jongh and A. Visser.

The main purpose of this paper is to construct explicit bases of admissible rules for a class of modal logics extending $K 4$. The general outline of the completeness proof follows the method of R. Iemhoff; one of the differences lies in the choice of the basic notion - rather than dealing with admissible rules directly, we prefer to work with generalized rules

$$
\frac{\varphi_{1}, \ldots, \varphi_{k}}{\psi_{1}, \ldots, \psi_{\ell}} .
$$

There are several reasons why we consider this notion preferable. Intuitively speaking, the most remarkable and most important admissible rule of logics like $S 4, G L$, or $I P C$, is the disjunction property; the expressive power of the formal definition of an admissible rule should reflect this intuition. The other reasons are more technical: bases of admissible rules tend to look simpler and more natural when formulated as generalized rules, and the method we use in section 3 to prove Kripke completeness is better suited to generalized rules. The study of admissible multiple conclusion rules was already suggested in [17].

The material is organized as follows. Section 1 reviews basic notions and facts about normal modal logics and Kripke semantics, mainly to fix the notation; it also includes the necessary background from Ghilardi [11]. In section 2 we define the concept of $A R$-systems as the formal syntactical counterpart to sets of admissible rules, and we describe an embedding of $A R$-systems in quasi-normal logics. The technical core of the paper is section 3 , where we introduce specific $A R$-systems corresponding to admissible rules of the logics we have in mind, and we prove completeness of these $A R$-systems with respect to suitable Kripke semantics. The main results on bases of admissible rules are presented in section 4 . Section 5 briefly discusses two questions on sets of admissible rules, namely their computational complexity, and maximality. Finally, in section 6 we transfer the results of section 4 to bases of usual (single conclusion) admissible rules.

\section{Preliminaries}

The modal language consists of propositional variables (a.k.a. atoms) $p_{i}$, usual propositional connectives, and the unary modal connective $\square$. We introduce the abbreviations $\nabla \varphi=\neg \square \neg \varphi$, $\bullet \varphi=\varphi \wedge \square \varphi, \diamond \varphi=\neg \odot \neg \varphi=\varphi \vee \diamond \varphi$, and $\square^{n} \varphi=\square \cdots \square \varphi$ (with $n$ boxes). A normal modal logic is a set $L$ of modal formulas which is closed under substitution, contains all propositional tautologies and the schema

$$
\square(\varphi \rightarrow \psi) \rightarrow(\square \varphi \rightarrow \square \psi)
$$

and is closed under the detachment and necessitation rules

$$
\begin{aligned}
\varphi, \varphi \rightarrow \psi & \vdash \psi, \\
\varphi & \vdash \varphi .
\end{aligned}
$$


The minimal normal modal logic is denoted by $K . K 4$ is its extension by the schema

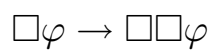

We will not list here axioms of other modal systems which appear in this paper; in case of doubt, the reader may consult e.g. [5].

If $L$ is a normal modal logic, we write $\vdash_{L} \varphi$ (or, occasionally, $L \vdash \varphi$ ) for $\varphi \in L$. More generally, if $\Gamma$ is a set of formulas, $\Gamma \vdash_{L} \varphi$ means that $\varphi$ is provable from $\Gamma$ using axioms and rules of $L$. (In particular, $\varphi \vdash_{L} \square \varphi$; we do not restrict the symbol $\vdash_{L}$ to the local consequence relation.) If $L$ contains $K 4$, we have the following version of the deduction theorem:

$$
\Gamma, \varphi \vdash_{L} \psi \quad \text { iff } \quad \Gamma \vdash_{L} \sqcup \varphi \rightarrow \psi
$$

A unifier for a formula $\varphi(\vec{p})$ is a substitution $\vec{\sigma}$ such that $\vdash_{L} \varphi(\vec{\sigma})$. (We denote substitutions by vectors of formulas; if we write $\vec{\sigma}$, it is understood that $\sigma_{i}$ is the formula to be substituted for the atom $p_{i}$.) A rule

$$
\frac{\varphi_{1}, \ldots, \varphi_{k}}{\psi}
$$

is $L$-admissible, written as $\varphi_{1}, \ldots, \varphi_{k} \sim_{L} \psi$, if every substitution which unifies all $\varphi_{1}, \ldots, \varphi_{k}$ also unifies $\psi$. A generalized rule consists of two finite sets of formulas, depicted as

$$
\frac{\varphi_{1}, \ldots, \varphi_{k}}{\psi_{1}, \ldots, \psi_{\ell}}
$$

Such a generalized rule is $L$-admissible $\left(\varphi_{1}, \ldots, \varphi_{k} \sim_{L} \psi_{1}, \ldots, \psi_{\ell}\right)$, if every substitution which unifies all $\varphi_{1}, \ldots, \varphi_{k}$ is also a unifier for some $\psi_{j}$. (Both $k$ and $\ell$ can be 0 ; for example, $\sim_{L}$ never holds, and $\perp \sim_{L}$ iff $L$ is consistent.) A generalized $L$-admissible rule $\Gamma \sim_{L} \Delta$ is derivable, if $\Gamma \vdash_{L} \psi$ for some $\psi \in \Delta \cup\{\perp\}$.

A Kripke frame is a pair $\langle K,<\rangle$, where $<$ is a binary relation on a non-empty set $K$. As we deal with extensions of $K 4$, we generally assume all frames to be transitive. (For the same reason, we will usually denote accessibility relations by the ordering symbol $<$. Beware: this notation does not mean that the relation $<$ is irreflexive.) A node $x \in K$ is reflexive, if $x<x$, otherwise it is irreflexive. The reflexivization $\leq$ of the accessibility relation is defined as $x \leq y$ iff $x<y$ or $x=y$. As $\leq$ is a preorder, it induces an equivalence relation $x \approx y$ iff $x \leq y$ and $y \leq x$. The cluster of a node $x$ is $\operatorname{cl}(x)=\{y ; x \approx y\}$. Notice that $\operatorname{cl}(x)=\{x\}$ whenever $x$ is irreflexive. The subframe generated by $x$ is $K_{x}=\{y ; x \leq y\}$. A rooted frame is a triple $\langle K,<, r\rangle$, where $\langle K,<\rangle$ is a frame, and $r \in K$ is such that $K=K_{r}$. A bisimulation of frames $\langle K,<\rangle$ and $\left\langle K^{\prime},<^{\prime}\right\rangle$ is a binary relation $B \subseteq K \times K^{\prime}$ such that

- if $x B x^{\prime}$ and $x<y$, there is $y^{\prime}$ such that $x^{\prime}<^{\prime} y^{\prime}$ and $y B y^{\prime}$,

- if $x B x^{\prime}$ and $x^{\prime}<^{\prime} y^{\prime}$, there is $y$ such that $x<y$ and $y B y^{\prime}$.

A $p$-morphism is an onto function $f: K \rightarrow K^{\prime}$ such that the graph of $f$ is a bisimulation. Bisimulations (hence also p-morphisms) of rooted frames $\langle K,<, r\rangle,\left\langle K^{\prime},<^{\prime}, r^{\prime}\right\rangle$ are additionally required to satisfy $r B r^{\prime}$. 
A Kripke model is a triple $\langle K,<, \Vdash\rangle$, where $\langle K,<\rangle$ is a frame, and $\Vdash$ is a satisfaction relation between $K$ and the set of propositional variables. We extend $\Vdash$ to all formulas using the usual rules for propositional connectives, and

$$
x \Vdash \square \varphi \quad \text { iff } \quad \forall y>x y \Vdash \varphi .
$$

The notions of generated and rooted models are defined similarly as for frames. A formula $\varphi$ is satisfied in a rooted model $\langle K,<, r, \Vdash\rangle$ if $r \Vdash \varphi$, and it is satisfied in a model $\langle K,<, \Vdash\rangle$ if $x \Vdash \varphi$ for all $x \in K . \varphi$ is valid in a frame or rooted frame, if it is satisfied in every (rooted) model based on this frame. Generated submodels preserve satisfaction. As a consequence, a formula is valid in a frame $\langle K,<\rangle$ iff it is valid in all its generated subframes.

Bisimulations and p-morphisms of (rooted) models are defined as for frames, with the additional condition

- if $x B x^{\prime}$, then $x \Vdash p$ iff $x^{\prime} \Vdash p$ for every atom $p$.

It follows that bisimulations of models preserve satisfaction of all formulas, and p-morphisms of frames preserve validity. If $\langle K,<, r, \Vdash\rangle$ and $\left\langle K^{\prime},<^{\prime}, r^{\prime}, \Vdash^{\prime}\right\rangle$ are rooted models, we write $K_{r} \sim K_{r^{\prime}}^{\prime}$ if there exists a bisimulation of $\langle K,<, r, \Vdash\rangle$ and $\left\langle K^{\prime},<^{\prime}, r^{\prime}, \Vdash^{\prime}\right\rangle$.

Let $L$ be a normal modal logic. An $L$-frame is a frame which validates all theorems of $L$. A (rooted) $L$-model is a (rooted) model based on an $L$-frame. The logic $L$ is complete, if every formula $\varphi$ which is valid in all $L$-frames is derivable in $L . L$ has the finite model property (FMP), if every formula valid in all finite $L$-frames is derivable.

If $L$ has FMP, we define $\operatorname{Mod}_{L}(\varphi)$ as the set of all finite rooted $L$-models which satisfy $\varphi$. $M O D_{L}(\varphi)$ is the set of finite rooted $L$-models $\langle K,<, r, \Vdash\rangle$ such that $\varphi$ is satisfied in the model $\langle K,<, \Vdash\rangle$; i.e., $\operatorname{MOD}_{L}(\varphi)=\operatorname{Mod}_{L}(\square \varphi)$.

The box-depth $d(\varphi)$ of a formula $\varphi$ is the maximal number of nested boxes in $\varphi$, i.e., $d\left(p_{i}\right)=0, d(\varphi \circ \psi)=\max \{d(\varphi), d(\psi)\}$ if $\circ$ is a propositional connective, and $d(\square \varphi)=1+d(\varphi)$. If $\Gamma$ is a finite set of formulas, we put $d(\Gamma)=\max \{d(\varphi) ; \varphi \in \Gamma\}$.

Fine $[7,8]$ introduced approximations $\sim_{n}$ to the bisimulation relation. We define $\sim_{n}$ by induction on $n$ :

- $K_{x} \sim_{0} K_{x^{\prime}}^{\prime}$ iff $x$ and $x^{\prime}$ satisfy the same propositional variables,

- $K_{x} \sim_{n+1} K_{x^{\prime}}^{\prime}$ iff $K_{x} \sim_{0} K_{x^{\prime}}^{\prime}, \forall y>x \exists y^{\prime}>x^{\prime} K_{y} \sim_{n} K_{y^{\prime}}^{\prime}$, and vice versa.

All $\sim_{n}$ are equivalence relations, and $\sim_{0} \supseteq \sim_{1} \supseteq \sim_{2} \supseteq \cdots \supseteq \sim$; for finite models, we have $K_{x} \sim K_{x^{\prime}}^{\prime}$ iff $\forall n K_{x} \sim_{n} K_{x^{\prime}}^{\prime}$. The importance of the relations $\sim_{n}$ is explained by the following result.

1.1 Proposition (Fine) Let $M$ be a set of finite rooted $L$-models in a finite number of variables. Then $M$ is closed under $\sim_{n}$ iff $M=\operatorname{Mod}_{L}(\varphi)$ for some $\varphi$ of depth $d(\varphi) \leq n$.

In particular, $\sim_{n}$ preserves satisfaction of formulas of box-depth at most $n$.

Now we introduce the fundamental result of S. Ghilardi on projective formulas. Let $L$ be a normal extension of $K 4$ with FMP. A formula $\varphi(\vec{p})$ is $L$-projective, if there is a substitution $\vec{\chi}$ such that 
$(i) \vdash_{L} \varphi(\vec{\chi})$,

(ii) $\varphi \vdash_{L} p_{i} \equiv \chi_{i}$ for every atom $p_{i}$.

$\vec{\chi}$ is the projective substitution for $\varphi$.

A class $M$ of finite rooted $L$-models has the $L$-extension property, if it is closed under isomorphism, and the following holds for any rooted $L$-model $\langle K,<, r, \Vdash\rangle$ with $|\operatorname{cl}(r)|=1$ : if $K_{x} \in M$ for every $x \neq r$, then there exists $K^{\prime} \in M$ identical to $K$ except for satisfaction of atoms in $r$.

1.2 Theorem (Ghilardi [11]) Let $L$ be a normal extension of $K 4$ with FMP. A formula $\varphi$ is $L$-projective if and only if $M O D(\varphi)$ has $L$-extension property.

\section{$2 \quad A R$-systems}

We introduce the notion of an $A R$-system as the syntactical framework in which we will investigate the admissibility relation $\Gamma \sim \Delta$. This also clarifies what we mean by a basis of admissible generalized rules. (The usual definition of a basis for single-conclusion rules based on composition does not make nearly as much sense for the generalized rules.)

2.1 Definition Let $L$ be a normal extension of $K 4$. An $A R$-system over $L$ is a set $A$ of sequents of the form $\Gamma \triangleright \Delta$ (where $\Gamma$ and $\Delta$ are finite sets of formulas), which is closed under cut, weakening, and substitution, and contains all sequents

$$
\Gamma \triangleright \varphi
$$

such that $\Gamma \vdash_{L} \varphi$. We identify $L$ with the minimal $A R$-system over $L$. A set $X$ of sequents axiomatizes $A$ over $L$, if $A$ is the smallest $A R$-system over $L$ which contains $X$. (We will generally assume that $X$ is already closed under substitution.)

The set of all $L$-admissible generalized rules is clearly an $A R$-system; its axiomatization over $L$ is called a basis.

A model $\langle K,<, \Vdash\rangle$ satisfies an $A R$-sequent $\Gamma \triangleright \Delta$, if it satisfies some formula from $\Delta$, or if it does not satisfy all formulas from $\Gamma$. $\Gamma \triangleright \Delta$ is valid in a frame $\langle K,<\rangle$, if it is satisfied in all models based on $\langle K,<\rangle$.

If $\Gamma$ is a set of formulas, we put $\square \Gamma=\{\square \varphi ; \varphi \in \Gamma\}, \bigwedge \Gamma=\bigwedge_{\varphi \in \Gamma} \varphi$, and $\bigvee \Gamma=\bigvee_{\varphi \in \Gamma} \varphi$ The characteristic formula of the sequent $\Gamma \triangleright \Delta$ is

$$
\bigwedge \square \Gamma \rightarrow \bigvee \square \Delta
$$

If $X$ is a set of $A R$-sequents axiomatizing an $A R$-system $A$ over $L$, we define $A^{\square}$ to be the modal logic axiomatized by the detachment rule, theorems of $K 4, \square L$, and (substitution instances of) characteristic formulas of sequents from $X$. (In general, $A^{\square}$ is not a normal logic; however notice that $L^{\square}$ is always normal.)

A rooted frame $\langle K,<, r\rangle$ is an $L^{\square}$-frame, if $<$ is transitive, $r$ is irreflexive, and $K \backslash\{r\}$ is an $L$-frame. (Notice that $\Gamma \triangleright \Delta$ is valid in $K \backslash\{r\}$ iff the characteristic formula of $\Gamma \triangleright \Delta$ is valid in $K$.) 
The representation of $A R$-systems by the logics $A^{\square}$ is a convenient way to avoid development of ad hoc semantical methods for such systems. As we will see shortly, this embedding is faithful, due to the free-cut elimination theorem for classical propositional logic (cf. [3]):

2.2 Theorem Assume that a sequent $\Gamma \Longrightarrow \Delta$ follows from a set $X$ of sequents. Then there is a $G K$-proof $\pi$ of $\Gamma \Longrightarrow \Delta$ using extra initial sequents from $X$, such that every cut formula in $\pi$ has a direct ancestor in a sequent in $X$.

2.3 Lemma Let $A$ be an $A R$-system over $L$. Then $A^{\square}$ is conservative over $A$, i.e., a sequent $\Gamma \triangleright \Delta$ is provable in $A$ if and only if its characteristic formula is provable in $A^{\square}$.

Proof: The "only if" direction is easy, we will show the "if" direction. Let $G K A$ be the extension of the classical propositional Gentzen calculus $G K$ by initial sequents of the form

$$
\begin{aligned}
\square(\varphi \rightarrow \psi), \square \varphi & \Longrightarrow \square \psi, \\
\square \varphi & \Longrightarrow \square \square \varphi, \\
& \Longrightarrow \square \lambda, \\
\square \Gamma & \Longrightarrow \square \Delta,
\end{aligned}
$$

where $\vdash_{L} \lambda$, and $\Gamma \triangleright \Delta$ is any axiom of $A$. Clearly, $A^{\square}$ proves the characteristic formula of $\Gamma \triangleright \Delta$ iff $G K A$ proves the sequent $\square \Gamma \Longrightarrow \square \Delta$. If we consider boxed formulas as new propositional atoms for a while, we can apply theorem 2.2, and obtain a $G K A$-proof $\pi$ of $\square \Gamma \Longrightarrow \square \Delta$ such that each cut formula appears in an initial sequent. By the subformula property, every formula in $\pi$ is a (propositional) subformula of a formula from $\Gamma \cup \Delta$, or a cut formula. This implies that every formula in $\pi$ is boxed; in particular, $\pi$ cannot use any introduction rules for propositional connectives, the only derivations in $\pi$ are thus cuts and weakenings. (We consider sequents of $G K$ to consist of sets of formulas rather than sequences, thus we do not need exchange and contraction rules.) If we remove the boxes in front of all formulas, and change the sequent arrow to $\triangleright$, we get an $A$-proof of $\Gamma \triangleright \Delta$.

2.4 Lemma Let $L$ be a complete normal extension of $K 4$. Then $L^{\square}$ is sound and complete with respect to the class of $L^{\square}$-frames. If moreover $L$ has FMP, then $L^{\square}$ has FMP as well.

Proof: Soundness is straightforward. Assume that $L^{\square} \nvdash \varphi$, let $S$ be a finite set containing $\varphi$, and closed under negation and subformulas, and let $X \subseteq S$ be a maximal $L^{\square}$-consistent set such that $\neg \varphi \in X$. Put $Y=\{\psi ; \square \psi \in X\}$. For any $\psi$ such that $\neg \square \psi \in X$, the set $Y \cup \square Y \cup\{\neg \psi\}$ is $L$-consistent: otherwise we would have $L \vdash \bullet Y \rightarrow \psi$, thus $\square L \vdash \square(\square Y \rightarrow$ $\psi$ ), and $K 4+\square L \vdash \square Y \rightarrow \square \psi$, contradicting the consistency of $X$. By completeness of $L$, there exists a rooted $L$-model $\left\langle K_{\psi},<_{\psi}, r_{\psi}, \Vdash\right\rangle$ such that $r_{\psi} \nVdash \psi$, and $r_{\psi} \Vdash Y \cup \square Y$. We construct a new model $\langle K,<, r, \Vdash\rangle$ by taking the disjoint union of all $K_{\psi}$, and putting a new irreflexive root $r$ below all the other nodes. The forcing of atoms in $r$ is defined by

$$
r \Vdash p \quad \text { iff } \quad p \in X .
$$

We have that $<$ is transitive (each $<_{\psi}$ is transitive as $L \supseteq K 4$ ), and $K \backslash\{r\}$ is an $L$-frame (as $L$-frames are closed under taking disjoint unions). A straightforward induction on complexity 
shows that $r \Vdash X$, thus $r \nVdash \varphi$. If $L$ has FMP, we can take all $K_{\psi}$ to be finite, in which case $K$ is also finite.

\section{Extensible logics and models}

In this section we present the class of logics $L$ for which our methods naturally apply, formulate $A R$-systems $A_{L}$ (which, as we will see in the next section, characterize $L$-admissible generalized rules), and prove a Kripke completeness result for the corresponding non-normal modal systems $A_{L}^{\square}$.

3.1 Definition Let $L$ be a normal extension of $K 4$ with FMP. $L$ is reflexive, if all $L$-frames are reflexive (i.e., $L \supseteq S 4$ ), and it is irreflexive if all $L$-frames are irreflexive (i.e., $L \supseteq G L$ ). $L$ is linear if all rooted $L$-frames are linear (i.e., trichotomic), or equivalently, if $L$ proves the schema

$$
\square(\square \varphi \rightarrow \psi) \vee \square(\square \psi \rightarrow \varphi) .
$$

If $K_{i}, i<n$ are frames, then $\sum_{i<n} K_{i}$ is their disjoint sum. If $K$ is a frame, then $K^{\circ}\left(K^{\bullet}\right)$ is the frame constructed from $K$ by attaching a new reflexive (irreflexive) root below $K$. $L$ is extensible, if for every finite sequence of finite $L$-frames $K_{i}, i<n$, we have

- $\left(\sum_{i<n} K_{i}\right)^{\circ}$ is an $L$-frame, unless $L$ is irreflexive,

- $\left(\sum_{i<n} K_{i}\right)^{\bullet}$ is an $L$-frame, unless $L$ is reflexive.

$L$ is linear extensible, if it is linear, and satisfies the extensibility condition above for $n \leq 1$.

3.2 Definition Let $\langle K,<\rangle$ be an $L$-frame. A node $x$ is a tight predecessor of a finite set of nodes $\left\{y_{i} ; i<n\right\}$, if

$$
z>x \quad \text { iff } \quad \exists i z \geq y_{i}
$$

holds for every $z \in K$ distinct from $x$.

An $L$-frame $\langle K,<\rangle$ is extensible (resp., linearly extensible), provided $K_{x}$ is finite for every $x \in K$, and every finite subset of $K$ (resp., every subset of size at most 1 ) has a reflexive tight predecessor unless $L$ is irreflexive, and an irreflexive tight predecessor unless $L$ is reflexive.

The following lemma will be used in section 4 .

3.3 Lemma If $K$ is an extensible $L$-model, then the set of all finite rooted $L$-models $W$ such that $W \sim_{n} K_{y}$ for some $y \in K$ has $L$-extension property. The same holds for linearly extensible $K$, if the logic $L$ is linear.

Proof: Let $\langle W,<, z, \Vdash\rangle$ be a rooted $L$-model, $x_{i}(i<k)$ successors of $z$ such that $W \backslash\{z\}=$ $\bigcup_{i} W_{x_{i}}$, and $W_{x_{i}} \sim_{n} K_{y_{i}}$ for some $y_{i} \in K$. If $L$ is linear, we may assume $k \leq 1$. Let $y \in K$ be a tight predecessor of $\vec{y}$, reflexive or irreflexive according to the reflexivity of $z$, and modify the satisfaction of atoms in $z$ to agree with $y$. Put $W^{\prime}=\left(\sum_{i} W_{x_{i}}\right)^{*}$ and $K^{\prime}=\left(\sum_{i} K_{y_{i}}\right)^{*}$ with satisfaction inherited from $W$ and $K$, where $*$ is $\circ$ or $\bullet$ as appropriate. Then $W^{\prime} \sim_{n} K^{\prime}$ : if $z$ is irreflexive, it follows directly from the definition; if $z$ is reflexive, we use straightforward induction on $n$. Consequently $W \sim W^{\prime} \sim_{n} K^{\prime} \sim K$. 
3.4 Definition We introduce the following sets of $A R$-sequents:

$$
\begin{aligned}
& \square \varphi \rightarrow \bigvee_{i<n} \square \psi_{i} \triangleright\left\{\boxminus \varphi \rightarrow \psi_{i} ; i<n\right\} \\
& \bigwedge_{j<m}\left(\varphi_{j} \equiv \square \varphi_{j}\right) \rightarrow \bigvee_{i<n} \square \psi_{i} \triangleright\left\{\bigwedge_{j<m} \boxminus \varphi_{j} \rightarrow \psi_{i} ; i<n\right\}
\end{aligned}
$$

where $n, m \in \omega$. Let $A^{\circ, 1}$ and $A^{\bullet, 1}$ be the restrictions of $A^{\circ}$ and $A^{\bullet}$ to $n \leq 1$. If $L$ is extensible, we define $A_{L}$ as the $A R$-system axiomatized over $L$ by the sequents

- $A^{\bullet}$, unless $L$ is reflexive,

- $A^{\circ}$, unless $L$ is irreflexive.

If $L$ is linear extensible, we define $A_{L}$ similarly, using $A^{\circ, 1}$ and $A^{\bullet, 1}$.

The proof of completeness of $A_{L}^{\square}$ in the next theorem was inspired by the completeness proof for Japaridze's provability logic $D$ by L. Beklemishev [1] (the idea might have been already present in [16]).

3.5 Theorem If $L$ is extensible (linear extensible), then $A_{L}^{\square}$ is sound and complete with respect to rooted $L^{\square}$-frames $\langle K,<, r\rangle$ such that $K \backslash\{r\}$ is extensible (linearly extensible).

Proof: Assume first that $L$ is extensible.

Soundness: assume that $L$ is not reflexive, and $r \nVdash \bigvee_{i<n} \square\left(\boxminus \varphi \rightarrow \psi_{i}\right)$. Choose $y_{i}>r$ such that $y_{i} \Vdash \square \varphi \wedge \neg \psi_{i}$, and let $x>r$ be an irreflexive tight predecessor of $\left\{y_{i} ; i<n\right\}$. Then $x \Vdash \square \varphi$, but $x \nVdash \square \psi_{i}$ for any $i$, thus $r \nVdash \square\left(\square \varphi \rightarrow \bigvee_{i} \square \psi_{i}\right)$.

Assume that $L$ is not irreflexive, and $r \nVdash \bigvee_{i} \square\left(\bigwedge_{j} \boxminus \varphi_{j} \rightarrow \psi_{i}\right)$. As above, find $y_{i}>r$ such that $y_{i} \Vdash \bigwedge_{j} \sqcup \varphi_{j} \wedge \neg \psi_{i}$, and let $x>r$ be a reflexive tight predecessor of $\left\{y_{i} ; i<n\right\}$. Clearly $x \nVdash \square \psi_{i}$ for any $i$. Since $x$ is reflexive, and $\varphi_{j}$ holds in all nodes strictly above $x$, we have $x \Vdash \varphi_{j}$ iff $x \Vdash \square \varphi_{j}$, i.e., $x \Vdash \bigwedge_{j}\left(\varphi_{j} \equiv \square \varphi_{j}\right)$. Thus $r \nVdash \square\left(\bigwedge_{j}\left(\varphi_{j} \equiv \square \varphi_{j}\right) \rightarrow \bigvee_{i} \square \psi_{i}\right)$.

Completeness: assume $A_{L}^{\square} \nvdash \varphi$. By lemma 2.4, there exists a finite $L^{\square}$-model $\langle K,<, r, \Vdash\rangle$ such that $r \nVdash \varphi$, and $r \Vdash R_{\varphi}$, where $R_{\varphi}$ is the conjunction of all axioms of $A_{L}^{\square}$ of the form

$$
\square\left(\square \bigwedge_{j<m} \varphi_{j} \rightarrow \bigvee_{i<n} \square \psi_{i}\right) \rightarrow \bigvee_{i<n} \square\left(\square \bigwedge_{j<m} \varphi_{j} \rightarrow \psi_{i}\right)
$$

if $L$ is not reflexive, and

$$
\square\left(\bigwedge_{j<m}\left(\varphi_{j} \equiv \square \varphi_{j}\right) \rightarrow \bigvee_{i<n} \square \psi_{i}\right) \rightarrow \bigvee_{i<n} \square\left(\bigwedge_{j<m} \square \varphi_{j} \rightarrow \psi_{i}\right)
$$

if $L$ is not irreflexive, where $\square \varphi_{j}$ and $\square \psi_{i}$ are subformulas of $\varphi$.

Assume that $L$ is not reflexive, and let $y_{1}, \ldots, y_{k} \in K \backslash\{r\}$, we will show how to extend $K$ to a new model $K^{\prime}$, which contains an irreflexive tight predecessor of $Y=\left\{y_{1}, \ldots, y_{k}\right\}$. Let $\square \varphi_{j}, j<m$ be the list of all boxed subformulas of $\varphi$ such that $y_{\ell} \Vdash \square \varphi_{j}$ for all $\ell$. Let $\square \psi_{i}$, $i<n$ be the list of all remaining boxed subformulas of $\varphi$. We have $r \nVdash \bigvee_{i} \square\left(\square \bigwedge_{j} \varphi_{j} \rightarrow \psi_{i}\right)$, 
thus $r \nVdash \square\left(\square \bigwedge_{j} \varphi_{j} \rightarrow \bigvee_{i} \square \psi_{i}\right)$, and we can fix $w>r$ such that $w \Vdash \bigwedge_{j} \square \varphi_{j} \wedge \bigwedge_{i} \neg \square \psi_{i}$. Choose a new element $x$, and define $K^{\prime}=K \cup\{x\}$, where

$$
\begin{aligned}
& v<x \quad \text { iff } \quad v=r, \\
& x<v \text { iff } \exists \ell y_{\ell} \leq v, \\
& x \Vdash p \quad \text { iff } \quad w \Vdash p \text {. }
\end{aligned}
$$

If $\square \chi$ is a subformula of $\varphi$, we have $x \Vdash \square \chi$ iff $\forall \ell y_{\ell} \Vdash \square \chi$ iff $\chi$ is one of the $\varphi_{j}$ iff $w \Vdash \square \chi$. It follows by obvious induction that $x$ and $w$ agree on satisfaction of all subformulas of $\varphi$, and satisfaction of subformulas of $\varphi$ is preserved in the root $r$. Clearly, $x>r$ is an irreflexive tight predecessor of $Y$. Notice that $K^{\prime}$ is an $L^{\square}$-frame: it suffices to show that $K_{v}^{\prime}$ is an $L$-frame for every $v>r$. If $v \neq x$, then $K_{v}^{\prime}=K_{v} ; K_{x}^{\prime}$ is an $L$-frame as $L$ is extensible, and $K_{x}^{\prime}$ is a p-morphic image of $\left(\sum_{\ell} K_{y_{\ell}}\right)^{\bullet}$.

Also notice that $r \Vdash R_{\varphi}$ holds in $K^{\prime}$ : if $r \Vdash \square\left(\bigwedge_{j} \square \alpha_{j} \rightarrow \bigvee_{i} \square \beta_{i}\right)$ in $K^{\prime}$, then it also holds in $K$, thus (in $K$ ) there is $i$ such that $r \Vdash \square\left(\bigwedge_{j} \sqcup \alpha_{j} \rightarrow \beta_{i}\right)$. It follows that $v \Vdash \bigwedge_{j} \sqcup \alpha_{j} \rightarrow \beta_{i}$ in $K^{\prime}$ for all $v>r, v \neq x$. If $x \Vdash \bigwedge_{j} \sqcup \alpha_{j}$, then $w \Vdash \bigwedge_{j} \sqcup \alpha_{j}$ as this formula is a Boolean combination of subformulas of $\varphi$, thus $w \Vdash \beta_{i}$, and $x \Vdash \beta_{i}$.

If $L$ is not irreflexive, we can construct a reflexive tight predecessor of $Y$ in a similar way. The only difference is that we take $w \Vdash \bigwedge_{j}\left(\varphi_{j} \equiv \square \varphi_{j}\right) \wedge \bigwedge_{i} \neg \square \psi_{i}$. Again, $x$ and $w$ agree on satisfaction of subformulas of $\varphi$ : we show this by induction on complexity, the only interesting case being boxed subformulas $\square \chi$ of $\varphi$ : if $\chi=\psi_{i}$, we have $w \nVdash \square \psi_{i}$, and $x \nVdash \square \psi_{i}$. If $\chi=\varphi_{j}$, then $\varphi_{j}$ holds in all nodes strictly above $x$. Thus, $x \Vdash \square \varphi_{j}$ iff $x \Vdash \varphi_{j}$ iff (induction hypothesis) $w \Vdash \varphi_{j}$ iff $w \Vdash \square \varphi_{j}$, as $w \Vdash\left(\varphi_{j} \equiv \square \varphi_{j}\right)$. The rest of the argument is unchanged.

The remaining task is to repeat this construction so that tight predecessor are introduced for all finite sets of nodes. We can assume that the underlying set of $K \backslash\{r\}$ is $\{0, \ldots, m\}$ for some natural number $m$. We fix an enumeration $\left\{Y_{n} ; n \in \omega, n \geq m\right\}$ of finite subsets of $\omega$ such that all elements of $Y_{n}$ are smaller than $n$, and we construct a sequence of finite $L^{\square}$-models $K=K^{m} \subseteq K^{m+1} \subseteq K^{m+2} \subseteq \cdots$ with the following properties: $r$ is the root of all $K^{n}, K^{n} \supseteq\{0, \ldots, n\}, r \Vdash R_{\varphi}$ in $K^{n}$, satisfaction of subformulas of $\varphi$ is preserved in $r, K^{n+1}$ contains a reflexive and/or irreflexive tight predecessor of $Y_{n}$ where appropriate, and $K^{n+1}$ is an end-extension of $K^{n}$ (i.e., no new nodes are introduced in $K^{n+1}$ above any $v \in K^{n}$ except for $v=r)$.

We define $K^{\infty}=\bigcup_{n} K^{n}$. If $v \in K^{\infty} \backslash\{r\}$, then $K_{v}^{\infty}=K_{v}^{n}$ for some $n<\omega$ (namely, any $n$ such that $v \in K^{n}$ ), thus $K_{v}^{\infty}$ is finite. Also $K_{v}^{\infty}$ is an $L$-frame, thus $K^{\infty}$ is an $L^{\square}$-frame. Every finite subset of $K^{\infty} \backslash\{r\}$ has a reflexive and/or irreflexive tight predecessor, thus $K^{\infty} \backslash\{r\}$ is extensible. Finally, an obvious induction on complexity shows that forcing of subformulas of $\varphi$ is preserved in $r$, in particular $r \nVdash \varphi$ in $K^{\infty}$.

The proof for $L$ linear extensible is similar. In the soundness part, we simply demand $n \leq 1$. As for completeness, we redefine $R_{\varphi}$ as the conjunction of the formulas

$$
\begin{gathered}
\diamond \bigwedge_{j<m} \square \varphi_{j}, \\
\square\left(\square \bigwedge_{j<m} \varphi_{j} \rightarrow \bigvee_{i<n} \square \psi_{i}\right) \rightarrow \square\left(\triangleright \bigwedge_{j<m} \varphi_{j} \rightarrow \bigvee_{i<n} \square \psi_{i}\right),
\end{gathered}
$$


for $L$ not reflexive, and

$$
\begin{gathered}
\diamond \bigwedge_{j<m}\left(\varphi_{j} \equiv \square \varphi_{j}\right), \\
\square\left(\bigwedge_{j<m}\left(\varphi_{j} \equiv \square \varphi_{j}\right) \rightarrow \bigvee_{i<n} \square \psi_{i}\right) \rightarrow \square\left(\bigwedge_{j<m} \square \varphi_{j} \rightarrow \bigvee_{i<n} \square \psi_{i}\right),
\end{gathered}
$$

for $L$ not irreflexive, where $\varphi_{j}$ and $\psi_{i}$ are as before. $R_{\varphi}$ is provable in $A_{L}^{\square}$, as $\vdash \bigvee_{i} \square \psi_{i} \rightarrow$ $\square \bigvee_{i} \sqcup \psi_{i}$. The rest of the argument goes through, upto the fact that we deal only with sets $Y$ of size at most 1 .

\section{Bases of admissible generalized rules}

We derive the desired axiomatization of generalized admissible rules for extensible and linear extensible logics by combining results of section 3 with Ghilardi's work on unification [11]. Ghilardi's methods need certain adjustments to suit our present goal; this is incorporated in the proof of $(i) \rightarrow(v) \rightarrow(i v)$ below.

We remind that all extensible and linear extensible logics are, by definition, normal extensions of $K 4$ with FMP.

4.1 Theorem Let $L$ be an extensible or linear extensible logic, and $\Gamma, \Delta$ finite sets of formulas. Then the following are equivalent.

(i) $\Gamma \sim_{L} \Delta$,

(ii) $A_{L}$ proves $\Gamma \triangleright \Delta$,

(iii) $A_{L}^{\square}$ proves the characteristic formula of $\Gamma \triangleright \Delta$,

(iv) $\Gamma \triangleright \Delta$ is valid in all extensible (linearly extensible) $L$-frames,

$(v)$ for every $L$-projective formula $\pi$, if $\pi \vdash_{L} \Gamma$, then $\pi \vdash_{L} \psi$ for some $\psi \in \Delta$.

Moreover, in $(v)$, it suffices to consider only formulas $\pi$ such that $d(\pi) \leq d(\Gamma)$.

Proof: $\quad(i i) \rightarrow(i)$ : assume that $L$ is not reflexive, and $\nvdash_{L} \boxminus \varphi \rightarrow \psi_{i}$ for all $i<n$, where $n \leq 1$ if $L$ is linear. Then there exist finite rooted $L$-models $\left\langle K_{i},<, r_{i}, \Vdash\right\rangle$ such that $r_{i} \Vdash \square \varphi \wedge \neg \psi_{i}$. Since $L$ is extensible, $K=\left(\sum_{i} K_{i}\right)^{\bullet}$ is an $L$-model, and obviously $r \Vdash \square \varphi \wedge \bigwedge_{i} \neg \square \psi_{i}$, where $r$ is the new root of $K$. If $L$ is not irreflexive, then $L$ admits $A^{\circ}\left(A^{\circ, 1}\right)$ by a similar argument, observing once again that $r \Vdash \varphi_{j} \equiv \square \varphi_{j}$ if $r$ is reflexive and $x \Vdash \varphi_{j}$ for all $x>r, x \neq r$.

$($ ii $) \equiv($ iii $) \equiv(i v)$ follows from theorem 3.5 and lemma 2.3.

$(v) \rightarrow(i v)$ : let $K$ be an extensible $L$-model which satisfies $\Gamma$. Define $M_{n}=\{W ; \exists x \in$ $\left.K W \sim_{n} K_{x}\right\}$, where $n=d(\Gamma)$. By proposition 1.1, $M_{n}=\operatorname{Mod}(\pi)$ for some $\pi$ such that $d(\pi) \leq n$, and $M_{n}$ has $L$-extension property by lemma 3.3. Thus $M O D(\pi)$ has $L$-extension property as well, and $\pi$ is projective by theorem 1.2. Since $M_{n} \subseteq \operatorname{Mod}(\varphi)$ for every $\varphi \in \Gamma$, we have $\pi \vdash \Gamma$, thus $\pi \vdash \psi$ for some $\psi \in \Delta$. As $\left\{K_{x} ; x \in K\right\}$ is a subclass of $M_{n}, K$ satisfies $\pi$, and a fortiori $\psi$. 
$(i) \rightarrow(v)$ : let $\vec{\chi}$ be the projective unifier for $\pi$. We have $\vdash \pi(\vec{\chi})$, thus $\pi \vdash \Gamma$ implies $\vdash \varphi(\vec{\chi})$ for all $\varphi \in \Gamma$. From $\Gamma \sim_{L} \Delta$ we get $\vdash \psi(\vec{\chi})$ for some $\psi \in \Delta$. As $\pi \vdash \psi \equiv \psi(\vec{\chi})$, we have $\pi \vdash \psi$.

4.2 Definition For any formula $\varphi$, the L-projective approximation of $\varphi$, written as $\Pi_{\varphi}$, is the set of all $L$-projective formulas $\pi$ such that $d(\pi) \leq d(\varphi)$, and $\pi \vdash_{L} \varphi$. ( $\Pi_{\varphi}$ is finite up to provable equivalence.)

4.3 Corollary (Ghilardi [11]) If $L$ is an extensible or linear extensible logic, and $\varphi$ a formula, then $\varphi \sim_{L} \Pi_{\varphi}$.

Another way to state this corollary is that the inclusion of $\vdash_{L}$ in $\sim_{L}$ has a left adjoint. Let $\varphi^{*}=\bigvee \boxminus \Pi_{\varphi}$.

4.4 Corollary (Ghilardi [11]) If $L$ is extensible or linear extensible, then

$$
\varphi \sim_{L} \psi \quad \text { iff } \varphi^{*} \vdash_{L} \psi
$$

for any formulas $\varphi$ and $\psi$.

Following [15], we observe that projective approximations allow us to loosen the assumptions of theorem 4.1.

4.5 Theorem Let $A$ denote one of

- $K 4+A^{\circ}+A^{\bullet}$,

- $G L+A^{\bullet}$,

- $S 4+A^{\circ}$,

- $K 4.3+A^{\circ, 1}+A^{\bullet, 1}$,

- $G L .3+A^{\bullet}, 1$,

- $S 4.3+A^{\circ, 1}$.

If $L$ is a modal logic admitting $A$, then $A$ is a basis for $L$-admissible generalized rules.

Proof: For concreteness, let $A=K 4+A^{\circ}+A^{\bullet}$. Assume $\Gamma \sim_{L} \Delta$, and put $\varphi=\bigwedge \Gamma$. We have $\varphi \sim_{K 4} \Pi_{\varphi}$ by corollary 4.3, which means that $\Gamma \triangleright \Pi_{\varphi}$ is derivable in $A$, by theorem 4.1. Consider any $\pi \in \Pi_{\varphi}$. We have $\pi \vdash_{K 4} \varphi$, thus $\pi \vdash_{L} \psi$ for some $\psi \in \Delta$, as projectivity is preserved in extensions, and the proof of $(i) \rightarrow(v)$ in theorem 4.1 did not use any assumption on $L$. Therefore $\pi \triangleright \Delta$ is provable in $L$ (viewed as an $A R$-system). Using several cuts on $\Gamma \triangleright \Pi_{\varphi}$, we obtain a proof of $\Gamma \triangleright \Delta$ in $L+A$. 
4.6 Example $A^{\bullet}+A^{\circ}$ is a basis of generalized admissible rules for $K 4, K 4.1$, and $\mathrm{Grz}^{-}$. Here, .1 is the following variant of the McKinsey formula,

$$
\triangleright \diamond \varphi \rightarrow \diamond \square \varphi
$$

and $G r z^{-}$is the "non-reflexive Grzegorczyk" logic: it is axiomatized by

$$
\square(\square(\varphi \rightarrow \square \varphi) \rightarrow \varphi) \rightarrow \square \square \varphi,
$$

and complete wrt transitive frames $\langle K,<\rangle$ such that $<\backslash i d$ is converse well-founded.

$A^{\bullet}$ is a basis of $G L$-admissible generalized rules.

$A^{\circ}$ is a basis of generalized admissible rules of $S 4, S 4.1$, and $G r z$. In the presence of $\square \varphi \rightarrow \varphi$, the rule $A^{\circ}$ can be simplified to

$$
\bigwedge_{j<m}\left(\varphi_{j} \rightarrow \square \varphi_{j}\right) \rightarrow \bigvee_{i<n} \square \psi_{i} \triangleright\left\{\bigwedge_{j<m} \square \varphi_{j} \rightarrow \psi_{i} ; i<n\right\} .
$$

For $G r z$, we may further simplify the rule by fixing $m=1$.

We should note here that admissible rules of $S 4$ were already axiomatized by V. Rybakov; the basis given in [21] is more complicated, but (of course) can be seen to be equivalent to $A^{\circ}$ (or rather, its single-conclusion version $\widehat{A}^{\circ}$ from section 6 ).

To complete the discussion for (normal) provability logics, we also present bases of admissible rules for the systems $G L+\square^{n} \perp$.

4.7 Definition If $\langle K,<\rangle$ is a converse well-founded frame, and $x \in K$, we define the depth of $x$ by induction:

$$
d(x)=\sup \{d(y)+1 ; x<y\} .
$$

Notice that leaves have depth 0 , and for finite $n$ we have $d(x)=n$ iff $x \Vdash \square^{n+1} \perp \wedge \diamond^{n} \top$.

4.8 Theorem For any $k>0$, generalized admissible rules of $G L+\square^{k} \perp$ are axiomatized by $\left(A_{(k)}^{\bullet}\right)$

$$
\square \varphi \rightarrow \bigvee_{i<n} \psi_{i} \triangleright\left\{\square \varphi \wedge \square^{k-1} \perp \rightarrow \psi_{i} ; i<n\right\}
$$

Proof (sketch): Put $L=G L+\square^{k} \perp$. Clearly,

$$
\Gamma \sim_{L} \Delta \quad \text { iff } \quad\left\{\square^{k} \perp \rightarrow \varphi ; \varphi \in \Gamma\right\} \sim_{G L}\left\{\square^{k} \perp \rightarrow \psi ; \psi \in \Delta\right\},
$$

which immediately implies the admissibility of $A_{(k)}^{\bullet}$. Conversely, assume that $L+A_{(k)}^{\bullet}$ does not prove $\Gamma \triangleright \Delta$. As in the proof of theorem 3.5, construct a rooted $G L$-model $\langle K,<, r, \Vdash\rangle$ such that $r \Vdash \square^{k+1} \perp \wedge \wedge \square \Gamma \wedge \neg \bigvee \square \Delta$, which is extensible with respect to finite subsets $Y \subseteq K \backslash\{r\}$ such that $d(y)<k-1$ for every $y \in Y$. Enlarge $K$ into a new model $K^{\prime}$ by introducing all missing tight predecessors, with arbitrary satisfaction of variables. Then $K^{\prime}$ is extensible, and satisfaction of formulas of the form $\square\left(\square^{k} \perp \rightarrow \alpha\right)$ is preserved in $r$, thus

$$
r \Vdash \bigwedge_{\varphi \in \Gamma} \square\left(\square^{k} \perp \rightarrow \varphi\right) \wedge \neg \bigvee_{\psi \in \Delta} \square\left(\square^{k} \perp \rightarrow \psi\right)
$$

This implies $\left\{\square^{k} \perp \rightarrow \varphi ; \varphi \in \Gamma\right\} \not \mathscr{G}_{G L}\left\{\square^{k} \perp \rightarrow \psi ; \psi \in \Delta\right\}$ by theorem 4.1, thus $\Gamma \not_{L} \Delta$. 
We now look more closely at linear systems. Theorem 4.5 gives a basis of admissible rules for logics like $K 4.3$ or $G r z^{-} .3$; for extensions of $S 4.3$ and $G L .3$, we can obtain nicer results. Admissible rules of extensions of $S 4.3$ were already characterized by Rybakov [20], here we get an alternative proof.

4.9 Lemma The rule $\neg \bigwedge_{j<m}\left(\varphi_{j} \rightarrow \square \varphi_{j}\right) \sim \perp$ is admissible in any normal modal logic.

Proof: Assume $\vdash_{L} \neg \bigwedge_{j<m}\left(\varphi_{j} \rightarrow \square \varphi_{j}\right)$, we will show that $L$ is inconsistent. We have $\vdash_{L}$ $\neg \square \bigwedge_{j} \varphi_{j}$, thus $L$ extends $D=K+\diamond \top$. As $L$ is closed under substitution, we may assume that the formulas $\varphi_{j}$ do not contain any propositional variables. However, $D$ proves $T \equiv \square \top$ and $\perp \equiv \square \perp$, thus any closed formula $\varphi_{j}$ is in $D$ equivalent to $\perp$ or $T$. It follows that $\vdash_{D} \varphi_{j} \rightarrow \square \varphi_{j}$, thus $\vdash_{L} \perp$.

4.10 Theorem Let $L$ be a consistent extension of $S 4.3$.

(i) $\diamond \varphi, \diamond \neg \varphi \triangleright$ is a basis of $L$-admissible generalized rules.

(ii) If furthermore $L \supseteq S 4.1$, then all $L$-admissible generalized rules are derivable.

Proof: (i) The rule

$$
\bigwedge_{j<m}\left(\varphi_{j} \rightarrow \square \varphi_{j}\right) \rightarrow \square \psi \triangleright \bigwedge_{j<m} \square \varphi_{j} \rightarrow \psi
$$

is derivable in $S 4$, as $\vdash \square \varphi_{j} \rightarrow\left(\varphi_{j} \rightarrow \square \varphi_{j}\right)$, and $\vdash \square \psi \rightarrow \psi$. Combining this with lemma 4.9 , and the observation in example 4.6, we see that every consistent extension of $S 4$ admits $A^{\circ, 1}$. By theorem $4.5, A^{\circ, 1}$ is a basis of admissible rules, as we assume $L \supseteq S 4$.3. We may exclude the derivable rule above from the basis, which leaves just $\neg \bigwedge_{j<m}\left(\varphi_{j} \rightarrow \square \varphi_{j}\right) \triangleright$. It thus suffices to simulate this rule using $\diamond \varphi, \diamond \neg \varphi \triangleright$. Define

$$
\psi=\bigwedge_{i<m}\left(\bigwedge_{j<i} \square\left(\varphi_{j} \rightarrow \square \varphi_{j}\right) \rightarrow\left(\varphi_{i} \rightarrow \square \varphi_{i}\right)\right),
$$

we will show $\neg \bigwedge_{i}\left(\varphi \rightarrow \square \varphi_{i}\right) \vdash \diamond \psi \wedge \diamond \neg \psi$. Clearly,

$$
\begin{aligned}
\vdash_{S 4} \square \psi \rightarrow \bigwedge_{i<m} \square\left(\bigwedge_{j<i} \square\left(\varphi_{j} \rightarrow \square \varphi_{j}\right) \rightarrow \square\left(\varphi_{i} \rightarrow \square \varphi_{i}\right)\right) \rightarrow & \\
& \rightarrow \square \bigwedge_{i<m}\left(\varphi_{i} \rightarrow \square \varphi_{i}\right) \rightarrow \diamond \bigwedge_{i<m}\left(\varphi_{i} \rightarrow \square \varphi_{i}\right) .
\end{aligned}
$$

We claim that

$$
\vdash_{S 4} \square \neg \psi \rightarrow \diamond \bigwedge_{i<m}\left(\varphi_{i} \rightarrow \square \varphi_{i}\right)
$$

holds as well. Consider any finite $S 4$-model $K$, and $x \in K$ such that $x \Vdash \square \neg \psi$. Pick a $y \geq x$ in a maximal cluster, and let $k<m$ be the smallest index such that $y \nVdash \square\left(\varphi_{k} \rightarrow \square \varphi_{k}\right)$. (If there is no such $k$, then $y \Vdash \bigwedge_{i}\left(\varphi_{i} \rightarrow \square \varphi_{i}\right)$, and we are finished.) As $y \nVdash \psi$, there is $i<m$ such that $y \Vdash \bigwedge_{j<i} \square\left(\varphi_{j} \rightarrow \square \varphi_{j}\right), y \Vdash \varphi_{i}$, and $y \nVdash \square \varphi_{i}$. Clearly, $i=k$, thus $y \Vdash \varphi_{k} \wedge \neg \square \varphi_{k}$. The definition of $k$ does not change, if we replace $y$ with any other element of its cluster; thus in fact $y \Vdash \square\left(\varphi_{k} \wedge \neg \square \varphi_{k}\right)$, which is a contradiction.

(ii) follows from $(i)$, as $S 4.1 \vdash \neg \square(\diamond \varphi \wedge \diamond \neg \varphi)$. 
4.11 Lemma If $G L .3 \nvdash \varphi(\vec{p})$, then there is a ground substitution $\vec{\chi}$ such that $\varphi(\vec{\chi}) \vdash_{G L .3}$ $\square^{n} \perp$ for some $n \in \omega$. If $G L .3+\square^{k} \perp \nvdash \varphi(\vec{p})$, we may further assume $n<k$.

Proof: Fix a model $M$ on the frame $\langle\{0, \ldots, n\},<\rangle$ such that $0 \nVdash \varphi$. Define $\chi_{i}=\bigvee_{x \Vdash p_{i}} \vartheta_{n-x}$, where $\vartheta_{x}=\square^{x+1} \perp \wedge \diamond^{x} \top$. By obvious induction on complexity of a formula $\psi$, we have $\vdash \vartheta_{n-x} \rightarrow \psi(\vec{\chi})$ whenever $x \Vdash \psi$, thus $\vdash \vartheta_{n} \rightarrow \neg \varphi(\vec{\chi})$. In other words, $\varphi(\vec{\chi}) \vdash \square^{n+1} \perp \rightarrow \square^{n} \perp$, thus $\varphi(\vec{\chi}) \vdash \square^{n} \perp$ by Löb's rule.

4.12 Corollary The only proper normal extensions of $G L .3$ are $G L .3+\square^{k} \perp$, for some $k \in \omega$.

4.13 Theorem Let $L$ be a consistent normal extension of $G L .3$. Generalized $L$-admissible rules are axiomatized by

$$
\perp \triangleright
$$

and either

if $L=G L .3$, or

$$
\square \varphi \triangleright \varphi
$$

$$
\square \varphi \triangleright \square^{k-1} \perp \rightarrow \varphi
$$

if $L=G L .3+\square^{k} \perp$.

Proof: First consider $L=G L .3$. By theorem 4.5, its admissible rules are axiomatizable by $A^{\bullet}, 1$, i.e.,

$$
\begin{gathered}
\neg \square \varphi \triangleright, \\
\square \varphi \rightarrow \square \psi \triangleright \boxminus \varphi \rightarrow \psi .
\end{gathered}
$$

The first rule is equivalent to $\perp \triangleright$, as $\neg \square \varphi \triangleright \triangleright \neg \square \varphi \triangleright \perp$ is derivable in $G L$, and the second one is equivalent to $\square \varphi \triangleright \varphi$, as GL.3 proves

$$
\bullet(\square \varphi \rightarrow \square \psi) \rightarrow \square(\bullet \varphi \rightarrow \psi) .
$$

Let $L=G L .3+\square^{k} \perp$, and $A=L+\perp \triangleright+\square \varphi \triangleright \square^{k-1} \perp \rightarrow \varphi$. For any $i<k, A$ proves

$$
\square^{i} \perp \vee \boxminus \psi \triangleright \psi
$$

This follows by induction on $i$ : the base case $i=0$ is trivial. The induction step: we have $\square^{i+1} \perp \vee \sqcup \psi \vdash \square\left(\square^{i} \perp \vee \boxminus \psi\right) \triangleright \square^{k-1} \perp \rightarrow \square \square^{i} \perp \vee \square \psi$. Since $\square^{i+1} \perp \vee \square \psi \vdash \square^{k-1} \perp \vee \square \psi$, we get $\square^{i+1} \perp \vee \boxminus \psi \triangleright \square^{i} \perp \vee \boxminus \psi$.

We claim

$$
\varphi \sim_{L} \psi \quad \text { iff } \quad \varphi \vdash_{L} \square^{k-1} \perp \vee \boxminus \psi
$$

The right-to-left implication follows from $\square^{k-1} \perp \vee \square \psi \sim \psi$, which we just established. Assume $\nvdash_{L} \sqcup \varphi \rightarrow \square^{k-1} \perp \vee \boxminus \psi$, and fix a finite $L$-model $K$ such that $x \Vdash \bullet \varphi, x \nVdash \square^{k-1} \perp$, and $x \nVdash \boxminus \psi$. Let $\vartheta_{i}$ and $\vec{\chi}$ be as in the proof of lemma 4.11. We have

$$
G L .3 \vdash \bigvee_{i<k} \vartheta_{i} \rightarrow \varphi(\vec{\chi}),
$$


as $x \Vdash \bullet \varphi$, and $d(x)=k-1$. Since $\bigvee_{i<k} \vartheta_{i} \equiv \square^{k} \perp$, we get $\vdash_{L} \varphi(\vec{\chi})$. On the other hand, $y \nVdash \psi$ for some $y \geq x$, thus

$$
G L .3 \vdash \psi(\vec{\chi}) \rightarrow \neg \vartheta_{i}
$$

for some $i<k$. If $\psi(\vec{\chi})$ were provable in $L$, we would have $\vdash_{L} \square^{i+1} \perp \rightarrow \square^{i} \perp$, thus $\vdash_{L} \square^{i} \perp$ by Löb's rule, which is impossible. Therefore $\varphi \not{ }_{L} \psi$.

The claim immediately implies that $A$ proves all $L$-admissible rules of the form $\Gamma \triangleright \Delta$, where $|\Delta| \leq 1$, it thus suffices to show that all $L$-admissible generalized rules are essentially single-conclusion. As in theorem 4.8, we have

$$
\Gamma \sim_{L} \Delta \quad \text { iff } \quad\left\{\square^{k} \perp \rightarrow \varphi ; \varphi \in \Gamma\right\} \sim_{G L .3}\left\{\square^{k} \perp \rightarrow \psi ; \psi \in \Delta\right\} .
$$

By the first part of the proof, GL.3-admissible generalized rules have a single-conclusion basis. It follows by induction on the length of derivation that for each $G L .3$-admissible rule $\Gamma \sim \Delta$, there is $\psi \in \Delta \cup\{\perp\}$ such that $\Gamma \sim \psi$; by $(*), L$ shares this property as well.

\section{$5 \quad$ Further properties}

Decidability of admissibility in many modal systems was established by Rybakov (see [20]) and Ghilardi [11]; the latter result immediately generalizes to the following proposition.

5.1 Proposition If $L$ is a decidable logic meeting requirements of theorem 4.5, then the set of $L$-admissible generalized rules is decidable.

Proof: By the proof of theorem 4.5, we have $\Gamma \sim_{L} \Delta$ iff $\forall \pi \in \Pi_{\Gamma} \exists \psi \in \Delta \pi \vdash_{L} \psi$, where $\Pi_{\Gamma}$ is the projective approximation of $\bigwedge \Gamma$ in a base logic $(K 4, G L, S 4, K 4.3, G L .3$, or $S 4.3)$. Projective approximations are effectively computable, as projectivity is decidable [11].

For concrete modal systems, we can present more efficient algorithms.

5.2 Theorem The set of generalized $L$-admissible rules is

(i) in ESPACE, for $L=K 4, G L, S 4, G r z^{-}$, or Grz,

(ii) in coNE, for $L=K 4.3, G r z^{-} .3$, or $G L+\square^{k} \perp$,

(iii) coNP-complete, for any consistent extension L of S4.3 or GL.3.

Proof: (i): by theorem 4.1 and lemma 2.3, it suffices to show that $A_{L}^{\square} \in E S P A C E$. The proof of theorem 3.5 shows that the mapping $\varphi \mapsto\left(R_{\varphi} \rightarrow \varphi\right)$ is an exponential-time reduction of $A_{L}^{\square}$ to $L^{\square}$, and the proof of lemma 2.4 implies that $L^{\square} \in \operatorname{coNP} P^{L}$ (to show that $L^{\square} \nvdash \varphi$, nondeterministically guess an evaluation of subformulas of $\varphi$ in the root of a hypothetical model, and use the decision procedure for $L$ to verify that the guess is sound). Since $L$ is in PSPACE (cf. [18, 4]), coNP $\subseteq$ PSPACE.

(ii): $L \in \operatorname{coNP}$, thus the argument we gave for $(i)$ shows $A_{L}^{\square} \in \operatorname{coN} E^{N P}$. We get rid of the extra quantifier by observing that all oracle calls in the $c o N P^{L}$ algorithm for $L^{\square}$ are positive, thus in fact $L^{\square} \in \operatorname{coNP}$, and $A_{L}^{\square} \in \operatorname{coNE}$. 
(iii): for $L=G L .3+\square^{k} \perp$, the proof of theorem 4.13 gives a p-time $d t t$-reduction of $L$-admissibility to $L$-derivability. This method can be adapted to obtain a similar characterization for $G L .3$ :

$$
\varphi \sim_{G L .3} \psi \quad \text { iff } \quad \varphi \vdash_{G L .3} \square^{n} \psi,
$$

where $n$ is the number of boxed subformulas of $\varphi$.

Let $L$ be a consistent extension of $S 4.3$. By theorem 4.10, generalized admissible rules of $L$ have a basis consisting of $A R$-sequents with empty succedent; it follows by straightforward induction that

$$
\Gamma \sim_{L} \Delta \quad \text { iff } \quad \Gamma \sim_{L} \text { or } \exists \psi \in \Delta \Gamma \vdash_{L} \psi .
$$

The second disjunct is coNP-testable, as $L \in \operatorname{coNP}$ by a result of E. Spaan [22]. As for the first one, we claim

$$
\varphi \sim_{L} \quad \text { iff } \quad \vdash_{\text {Max }} \neg \varphi,
$$

where $M a x$ is the logic axiomatized by $\psi \equiv \square \psi$. (I.e., a formula $\varphi$ is $L$-unifiable iff it is satisfiable in the one-element reflexive frame). On one hand, if $\vdash_{L} \varphi(\vec{\chi})$, then also $\vdash_{\operatorname{Max}} \varphi(\vec{\chi})$ as $L \subseteq \operatorname{Max}$, thus $\nvdash_{\operatorname{Max}} \neg \varphi$. On the other hand, if $\nvdash_{\operatorname{Max}} \neg \varphi$, then $\vdash_{\operatorname{Max}} \varphi(\vec{\chi})$ for some ground substitution $\vec{\chi}$, as Max is essentially just the classical propositional logic. All variable-free formulas are equivalent to $\top$ or $\perp$ in $D \subseteq \operatorname{Max} \cap L$, thus $\vdash_{L} \varphi(\vec{\chi})$, and $\varphi \not_{L}$.

5.3 Question In cases $(i)$ and (ii) of theorem 5.2, we have an exponential gap between an upper bound (ESPACE, coNE) and a lower bound (PSPACE, coNP) on the complexity of admissibility. Can we improve the bounds?

Another natural question is the following: which logics are maximal with respect to admissibility? For example, $S 4$ is not maximal, as its proper extension $G r z$ admits every generalized rule admissible in $S 4$. On the other hand, $G L$ is maximal in this sense, due to the next theorem.

5.4 Theorem Let $L$ be a quasi-normal logic (i.e., an extension of $K$ closed under $M P$ and substitution), which admits $A^{\bullet}$. Then $L \subseteq G L$.

Proof: For any finite rooted $G L$-model $\langle M,<, x, \Vdash\rangle$ in a finite number of variables, we define its characteristic formula $\varphi_{x}$ by induction on its depth:

$$
\varphi_{x}=\bigwedge_{x \Vdash p} p \wedge \bigwedge_{x \nVdash p} \neg p \wedge \square \bigvee_{y>x} \varphi_{y} \wedge \bigwedge_{y>x} \nabla \varphi_{y}
$$

It follows by simple induction that

(i) $x \Vdash \varphi_{x}$,

(ii) if $x \Vdash \varphi$, then $\vdash_{K} \varphi_{x} \rightarrow \varphi$.

Assume that $\nvdash_{G L} \varphi$. Then there is a finite model $\langle M,<, x, \Vdash\rangle$ such that $x \nVdash \varphi$, which implies

$$
\vdash_{L} \varphi \rightarrow \neg \varphi_{x}
$$


It thus suffices to show that each characteristic formula $\varphi_{x}$ is consistent with $L$. We will proceed by induction on the depth of $x$. Assume for contradiction that $\vdash_{L} \neg \varphi_{x}$, i.e.,

$$
\vdash_{L} \bigwedge_{x \Vdash p} p \wedge \square \bigvee_{y>x} \varphi_{y} \rightarrow \bigvee_{y>x} \square \neg \varphi_{y} \vee \bigvee_{x \nVdash p} p
$$

Let $n$ be the depth of $x$. For any formula $\varphi$, let $\bar{\varphi}$ denote $\varphi$ with $\square^{n} \perp \rightarrow p$ substituted for atoms $p$ such that $x \Vdash p$, and $\square^{n} \perp \wedge p$ for atoms $p$ such that $x \nVdash p$. Since $L$ is closed under substitution, we get

$$
\vdash_{L} \neg \square^{n} \perp \rightarrow\left(\square \bigvee_{y>x} \bar{\varphi}_{y} \rightarrow \bigvee_{y>x} \square \neg \bar{\varphi}_{y}\right)
$$

i.e.,

$$
\vdash_{L} \square \bigvee_{y>x} \bar{\varphi}_{y} \rightarrow \bigvee_{y>x} \square \neg \bar{\varphi}_{y} \vee \square^{n} \perp
$$

Since $y \Vdash \square^{n} \perp$ for any $y>x$, we have $\vdash \bigvee_{y>x} \varphi_{y} \rightarrow \square^{n} \perp$. Also $\vdash \square^{n+1} \perp \rightarrow \square(\varphi \equiv \bar{\varphi})$ for any $\varphi$, thus

$$
\vdash_{L} \square \bigvee_{y>x} \varphi_{y} \rightarrow \bigvee_{y>x} \square \neg \varphi_{y} \vee \square^{n} \perp
$$

If $n=0$, then $\square^{n} \perp$ is $\perp$ and we can leave it out from the disjunction. If $n>0$, there is $y>x$ of depth $n-1$; then $y \nVdash \square^{n-1} \perp$, thus $\vdash \varphi_{y} \rightarrow \neg \square^{n-1} \perp$, and $\vdash \square^{n} \perp \rightarrow \square \neg \varphi_{y}$. In both cases, we get

$$
\vdash_{L} \square \bigvee_{y>x} \varphi_{y} \rightarrow \bigvee_{y>x} \square \neg \varphi_{y}
$$

By $A^{\bullet}$, there is $z>x$ such that

$$
\vdash_{L} \bullet \bigvee_{y>x} \varphi_{y} \rightarrow \neg \varphi_{z} .
$$

Since $z \Vdash \triangleright \bigvee_{y>x} \varphi_{y}$, we have $\vdash \varphi_{z} \rightarrow \boxminus \bigvee_{y>x} \varphi_{y}$. This implies $\vdash_{L} \neg \varphi_{z}$, which contradicts the induction hypothesis.

5.5 Corollary $G L$ is the only logic which admits all $G L$-admissible generalized rules.

A similar argument shows that this maximality property is shared by $G L+\square \square^{k} \perp$, and theorem 4.13 immediately implies it holds for all extensions of $G L .3$. On the other hand, theorem 4.10 shows that the only extension of $S 4.3$ maximal wrt admissibility is the logic $K+\varphi \equiv \square \varphi$. R. Iemhoff [14] proved maximality of intuitionistic logic; this result transfers to Grz by the Blok-Esakia theorem $[2,6]$.

5.6 Question Are $\mathrm{Grz}^{-}$or $\mathrm{Gr}^{-} .3$ maximal with respect to admissibility?

\section{Ordinary admissible rules}

Usual admissible rules are just generalized admissible rules $\Gamma \sim \Delta$ where $|\Delta|=1$. We will show how to construct a basis of single-conclusion admissible rules, once we have a basis for the generalized rules; we begin with the simple case of linear modal systems. 
6.1 Proposition Let $X$ be a set of single-conclusion rules, $Y$ a set of rules with empty conclusion, and $A$ the $A R$-system axiomatized by $X \cup Y$ over a logic $L$. Then

$$
X \cup\{\Gamma \triangleright \perp ; \Gamma \triangleright \in Y\}
$$

is a basis of single-conclusion rules provable in $A$.

Proof: By a straightforward induction on the length of derivation in $A$, we can show that for every rule $\Gamma \triangleright \Delta$ provable in $A$, there is $\psi \in \Delta \cup\{\perp\}$ such that $\Gamma \triangleright \psi$ is provable in the system axiomatized by $X \cup\{\Gamma \triangleright \perp ; \Gamma \triangleright \in Y\}$. The result follows as $\perp \triangleright \varphi$ is $L$-derivable.

6.2 Definition Disjunction property is the set of $A R$-sequents

$$
\bigvee_{i<n} \square \varphi_{i} \triangleright\left\{\varphi_{i} ; i<n\right\} .
$$

If $X$ is a set of sequents axiomatizing over $L$ an $A R$-system $A$, we define

$$
\widehat{X}=\{\square \bigwedge \Gamma \vee \square \alpha \triangleright \bigvee \square \Delta \vee \alpha ; \Gamma \triangleright \Delta \in X\},
$$

and we denote $\widehat{A}$ the system axiomatized by $\widehat{X}$ over $L$.

6.3 Lemma If $\widehat{A}$ proves $\Gamma \triangleright \varphi$, then it also proves $\square \wedge \Gamma \vee \boxminus \psi \triangleright \boxminus \varphi \vee \boxminus \psi$, for any formula $\psi$.

Proof: By induction on the length of derivation. If $\Gamma \triangleright \varphi$ is $L$-derivable, the other sequent is also derivable. For elements of $\widehat{X}$ we have

$$
\begin{aligned}
& \bullet(\square \bigwedge \Gamma \vee \square \alpha) \vee \boxminus \psi \triangleright \square \bigwedge \Gamma \vee \square(\square \alpha \vee \boxminus \psi) \\
& \triangleright \bigvee \boxminus \Delta \vee \boxminus \alpha \vee \boxminus \psi \\
& \triangleright \boxminus(\bigvee \boxminus \Delta \vee \alpha) \vee \boxminus \psi \text {. }
\end{aligned}
$$

The induction step for cut: we have

$$
\begin{aligned}
& \bullet \bigwedge \Gamma_{1} \vee \square \psi \triangleright \boxminus \chi \vee \square \psi \\
& \varpi\left(\bigwedge \Gamma_{2} \wedge \chi\right) \vee \sqcup \psi \triangleright \boxminus \varphi \vee \boxminus \psi
\end{aligned}
$$

by the induction hypothesis. We obtain

$$
\bullet \bigwedge \Gamma_{2} \vee \boxminus \psi, \boxminus \chi \vee \boxminus \psi \triangleright \boxminus \varphi \vee \boxminus \psi
$$

from the second sequent, thus

$$
\bullet \bigwedge \Gamma_{1} \vee \triangleright \psi, \bullet \bigwedge \Gamma_{2} \vee \boxminus \psi \triangleright \bullet \varphi \vee \triangleright \psi
$$

by cut with the first one, thus also

$$
\bullet \bigwedge\left(\Gamma_{1} \cup \Gamma_{2}\right) \vee \triangleright \psi \triangleright \vee \varphi \vee \boxminus \psi .
$$


6.4 Theorem Let $A$ be an $A R$-system axiomatized by $X$ over $L \supseteq K 4$, such that $A$ proves the disjunction property. Then $\widehat{A}$ proves the same single-conclusion rules as $A$.

Proof: It is easy to see that $A$ contains $\widehat{A}$, because of the disjunction property. For the reverse direction, it suffices to show that whenever $A$ proves $\Gamma \triangleright \Delta$, then $\widehat{A}$ proves $\Gamma \triangleright \bigvee \square \Delta$. We proceed by induction on the length of derivation. If $\Gamma \triangleright \Delta$ is $L$-derivable, then $\Gamma \triangleright \bigvee \vee \Delta$ is also derivable. If $\Gamma \triangleright \Delta \in X$, we have in $\widehat{A}$

$$
\Gamma \triangleright \square \bigwedge \Gamma \vee \square \perp \triangleright \bigvee \square \Delta \vee \perp \triangleright \bigvee \nabla \Delta
$$

The induction step for weakening is obvious. Cut: by induction hypothesis, we have

$$
\begin{gathered}
\Gamma_{1} \triangleright \bigvee \cdot \Delta_{1} \vee \sqcup \varphi \\
\Gamma_{2}, \varphi \triangleright \bigvee \cdot \Delta_{2}
\end{gathered}
$$

From the first sequent we obtain

$$
\Gamma_{1}, \Gamma_{2} \triangleright \bullet \bigvee \vee \Delta_{1} \vee \bullet\left(\bigwedge \Gamma_{2} \wedge \varphi\right)
$$

by $L$-derivable reasoning. Lemma 6.3 applied to the second sequent yields

$$
\bullet\left(\bigwedge \Gamma_{2} \wedge \varphi\right) \vee \bullet \bigvee \boxminus \Delta_{1} \triangleright \bullet \bigvee \boxminus \Delta_{2} \vee \boxminus \bigvee \boxminus \Delta_{1}
$$

thus

$$
\Gamma_{1}, \Gamma_{2} \triangleright \bigvee \square \Delta_{1} \vee \bigvee \square \Delta_{2}
$$

6.5 Corollary Let $A$ be one of

- $K 4+\widehat{A}^{\circ}+\widehat{A}^{\bullet}$

- $G L+\widehat{A}^{\bullet}$,

- $S 4+\widehat{A}^{\circ}$.

If a logic $L$ admits $A$, then $A$ is a basis of ordinary $L$-admissible rules.

Proof: Theorems 4.5 and 6.4 immediately imply the result for $K 4, G L$, and $S 4$. Then we proceed as in the proof of theorem 4.5, except that we use $\bigvee \vee \Pi_{\varphi}$ instead of $\Pi_{\varphi}$.

6.6 Remark Corollary 6.5 may be applicable even when theorem 4.5 is not. For example, the results of section 4 are insufficient to determine the generalized admissible rules of $K 4.2$, $S 4.2$, or Grz.2. However, Grz.2 is easily seen to admit $\widehat{A}^{\circ}$, thus $\widehat{A}^{\circ}$ is a basis of ordinary Grz.2-admissible rules by corollary 6.5. (This argument fails for $S 4.2$, as $S 4.2$ does not admit the rule $\square(\diamond \varphi \wedge \diamond \neg \varphi) \vee \square \alpha \triangleright \alpha$, admissible for $S 4$.)

In some cases, it is possible to characterize generalized admissible rules by their singleconclusion fragment. We say that a logic $L$ has essentially single-conclusion admissible rules, if for every $\Gamma \sim_{L} \Delta$ there is $\psi \in \Delta \cup\{\perp\}$ such that $\Gamma \sim_{L} \psi$. Clearly, if $L$ has essentially singleconclusion admissible rules, and $X$ is a basis of ordinary $L$-admissible rules, then $X \cup\{\perp \triangleright\}$ is a basis of $L$-admissible generalized rules. 
6.7 Definition Let $L$ be a modal logic. A substitution $\vec{\sigma}$ is less general than $\vec{\sigma}^{\prime}$, written as $\vec{\sigma} \leq \vec{\sigma}^{\prime}$, if there is a substitution $\vec{\tau}$ such that $\vdash_{L} \sigma_{i}^{\prime}(\vec{\tau}) \equiv \sigma_{i}$ for every $i$. The logic $L$ has filtering unification, if the set of unifiers of any formula is directed, i.e., for every formula $\varphi$ and every unifiers $\vec{\sigma}_{1}$ and $\vec{\sigma}_{2}$ of $\varphi$, there is a unifier $\vec{\sigma}$ of $\varphi$ such that $\vec{\sigma}_{1} \leq \vec{\sigma}$ and $\vec{\sigma}_{2} \leq \vec{\sigma}$.

6.8 Theorem (Ghilardi, Sacchetti [12]) An extension $L$ of $K 4$ has filtering unification if and only if $L \supseteq K 4.2$.

Here, in absence of reflexivity, we define .2 as the schema

$$
\diamond \bullet \varphi \rightarrow \square \diamond \varphi
$$

6.9 Observation If a logic $L$ has filtering unification, then its admissible rules are essentially single-conclusion.

Proof: Assume $\Gamma \not_{L} \psi$ for every $\psi \in \Delta \cup\{\perp\}$, and fix unifiers $\vec{\sigma}_{\psi}$ of $\Gamma$ such that $\nvdash \psi\left(\vec{\sigma}_{\psi}\right)$. As $L$ has filtering unification, there exists a unifier $\vec{\sigma}$ of $\Gamma$ more general than each $\vec{\sigma}_{\psi}$. Then $\nvdash_{L} \psi(\vec{\sigma})$ for every $\psi$, thus $\Gamma \nvdash_{L} \Delta$.

6.10 Example $\widehat{A}^{\circ} \cup\{\perp \triangleright\}$ is a basis of generalized admissible rules of Grz.2.

To complete the picture, we show a partial converse to 6.9 .

6.11 Theorem Let $L$ be a normal extension of $K 4.1$. If $L$ has essentially single-conclusion admissible rules, then it has filtering unification.

Proof: By 6.8, it suffices to show $L \supseteq K 4.2$.

Assume first that $\vdash_{L} \diamond \square \perp$ (i.e., $\square \diamond \top \rightarrow \square \perp$ ), and consider the rule

$$
\sqcup p \vee \square \neg p \triangleright \square \perp \rightarrow p, \square \perp \rightarrow \neg p .
$$

If the rule is admissible, it has a single-conclusion admissible subrule, which means that either $\square p \sim_{L} \square \perp \rightarrow \neg p$ or vice versa. If we substitute $\top$ for $p$, we get $\vdash_{L} \neg \square \perp$, thus $L$ is inconsistent (and, a fortiori, proves $K 4.2$ ).

If the rule is not admissible, there is a formula $\varphi$ such that

$$
\begin{gathered}
\vdash_{L} \boxminus \varphi \vee \square \neg \varphi, \\
\square \perp \nvdash \varphi, \\
\square \perp \nvdash \neg \varphi .
\end{gathered}
$$

This means that $\varphi$ and $\neg \varphi$ are satisfiable in the one-element irreflexive frame, thus there exist (ground) substitutions $\vec{\alpha}$ and $\vec{\beta}$ such that $\square \perp \vdash \varphi(\vec{\alpha})$ and $\square \perp \vdash \neg \varphi(\vec{\beta})$. Define a new substitution $\vec{\varepsilon}$ by

$$
\varepsilon_{i}=p ? \alpha_{i}: \beta_{i}
$$


where $\varphi ? \psi: \chi$ is a shorthand for $(\varphi \wedge \psi) \vee(\neg \varphi \wedge \chi)$. Then $\vdash p \wedge \square \perp \rightarrow(\psi(\vec{\varepsilon}) \equiv \psi(\vec{\alpha}))$ for any $\psi$, thus $\vdash p \wedge \square \perp \rightarrow \varphi(\vec{\varepsilon})$, and similarly, $\vdash \neg p \wedge \square \perp \rightarrow \neg \varphi(\vec{\varepsilon})$. We obtain

$$
\vdash \boxminus \varphi(\vec{\varepsilon}) \rightarrow \square(\square \perp \rightarrow p) \rightarrow \square \diamond p
$$

as $\vdash \diamond \square \perp$, and similarly $\vdash \square \neg \varphi(\vec{\varepsilon}) \rightarrow \boxminus \diamond \neg p$. Since $\vdash \boxminus \varphi \vee \boxminus \neg \varphi$, we get $\vdash \square \diamond \neg p \vee \boxminus \diamond p$, i.e., K4.2.

Next, assume $L \supseteq D$, and consider

$$
\bullet p \vee \odot \neg p \triangleright \diamond p, \diamond \neg p
$$

If the rule is admissible, we get $\vdash_{L} \diamond \perp$, i.e., $\vdash_{L} \perp$. Otherwise, there is $\varphi$ such that

$$
\begin{gathered}
\vdash_{L} \boxminus \varphi \vee \square \neg \varphi, \\
\nvdash \diamond \varphi, \\
\nvdash \diamond \neg \varphi .
\end{gathered}
$$

It follows that both $\varphi$ and $\neg \varphi$ are satisfiable in the one-element reflexive frame $\circ$ : its theory $T h(\circ)$ is axiomatizable by $K+\diamond \top+\sqcup \psi \vee \square \neg \psi$, and it follows by simple induction that $T h(\circ) \vdash \psi$ implies $K D 4.1 \vdash \diamond \psi$.

Thus, there are ground substitutions $\vec{\gamma}$ and $\vec{\delta}$, such that $T h(\circ) \vdash \varphi(\vec{\gamma}) \wedge \neg \varphi(\vec{\delta})$. Since every ground formula is decidable in $D$, we get $\vdash_{L} \varphi(\vec{\gamma}) \wedge \neg \varphi(\vec{\delta})$. We put

$$
\varepsilon_{i}=p ? \gamma_{i}: \delta_{i}
$$

As before, we have $\vdash \square p \rightarrow \varphi(\vec{\varepsilon})$ and $\vdash \square \neg p \rightarrow \neg \varphi(\vec{\varepsilon})$, thus $\vdash \square \varphi(\vec{\varepsilon}) \vee \square \neg \varphi(\vec{\varepsilon})$ implies $\vdash \bullet \diamond p \vee \odot \odot \neg p$.

Finally, we move to the case of general $L$. If the rule

$$
\square p \vee \boxminus \neg p \triangleright \square \perp \rightarrow p, \square \perp \rightarrow \neg p, \sqcup \diamond \top \rightarrow \diamond p, \triangleright \diamond \top \rightarrow \diamond \neg p
$$

is admissible, we get either $\vdash_{L} \diamond \top$ or $\vdash_{L} \diamond \square \perp$, and we are finished by the previous parts of the proof. If the rule is not admissible, there is $\varphi$ such that

$$
\begin{gathered}
\vdash_{L} \boxminus \varphi \vee \boxminus \neg \varphi, \\
\square \perp \nvdash_{L} \varphi, \\
\square \perp \nvdash_{L} \neg \varphi, \\
\diamond \top \nvdash_{L} \diamond \varphi, \\
\diamond \top \nvdash_{L} \diamond \neg \varphi .
\end{gathered}
$$

As before, we get substitutions $\vec{\alpha}, \vec{\beta}, \vec{\gamma}$, and $\vec{\delta}$, such that

$$
\begin{gathered}
\square \perp \vdash_{L} \varphi(\vec{\alpha}), \\
\square \perp \vdash_{L} \neg \varphi(\vec{\beta}), \\
\diamond \top \vdash_{L} \varphi(\vec{\gamma}), \\
\diamond \top \vdash_{L} \neg \varphi(\vec{\delta}) .
\end{gathered}
$$


We define $\varepsilon_{i}=\square \perp ?\left(p ? \alpha_{i}: \beta_{i}\right):\left(p ? \gamma_{i}: \delta_{i}\right)$. We obtain

$$
\begin{gathered}
\vdash_{L} p \wedge \square \perp \rightarrow \varphi(\vec{\varepsilon}), \\
\vdash_{L} \neg p \wedge \square \perp \rightarrow \neg \varphi(\vec{\varepsilon}), \\
\vdash_{L} \odot(p \wedge \diamond \top) \rightarrow \varphi(\vec{\varepsilon}), \\
\vdash_{L} \sqcup(\neg p \wedge \diamond \top) \rightarrow \neg \varphi(\vec{\varepsilon}),
\end{gathered}
$$

thus

$$
\vdash \boxminus \varphi(\vec{\varepsilon}) \rightarrow \boxminus(\square \perp \rightarrow p) \wedge \boxminus \diamond(\square \perp \vee p) \rightarrow \boxminus(\diamond \square \perp \rightarrow \diamond p) \wedge \boxminus(\square \diamond \top \rightarrow \diamond p) \rightarrow \boxminus \diamond p,
$$

and similarly $\vdash_{L} \sqcup \neg \varphi(\vec{\varepsilon}) \rightarrow \boxminus \diamond \neg p$, thus $\vdash_{L} \boxminus \diamond p \vee \boxminus \diamond \neg p$.

6.12 Question Does theorem 6.11 hold for arbitrary extensions of $K 4$ ?

\section{References}

[1] Lev D. Beklemishev, A provability logic without Craig's interpolation property, Matematicheskie Zametki 45 (1989), no. 6, pp. 12-22 (in Russian), English translation in: Mathematical Notes of the Academy of Sciences of the USSR 45 (1989).

[2] Willem J. Blok, Varieties of interior algebras, Ph.D. thesis, University of Amsterdam, 1976.

[3] Samuel R. Buss, An introduction to proof theory, in: Handbook of Proof Theory (S. R. Buss, ed.), Studies in Logic and the Foundations of Mathematics vol. 137, Elsevier, Amsterdam, 1998, pp. 1-78.

[4] Alexander V. Chagrov, On the complexity of propositional logics, in: Complexity problems in Mathematical Logic, Kalinin State University, 1985, pp. 80-90 (in Russian).

[5] Alexander V. Chagrov and Michael Zakharyaschev, Modal logic, Oxford Logic Guides vol. 35, Oxford University Press, 1997.

[6] Leo L. Esakia, To the theory of modal and superintuitionistic systems, in: Logical Inference. Proceedings of the USSR Symposium on the Theory of Logical Inference (V. A. Smirnov, ed.), Nauka, Moscow, 1979, pp. 147-172 (in Russian).

[7] Kit Fine, Logics containing K4, Part I, Journal of Symbolic Logic 39 (1974), no. 1, pp. $31-42$.

[8] Logics containing K4, Part II, Journal of Symbolic Logic 50 (1985), no. 3, pp. 619-651.

[9] Harvey M. Friedman, One hunderd and two problems in mathematical logic, Journal of Symbolic Logic 40 (1975), no. 2, pp. 113-129. 
[10] Silvio Ghilardi, Unification in intuitionistic logic, Journal of Symbolic Logic 64 (1999), no. 2 , pp. 859-880.

[11] Best solving modal equations, Annals of Pure and Applied Logic 102 (2000), no. 3, pp. 183-198.

[12] Silvio Ghilardi and Lorenzo Sacchetti, Filtering unification and most general unifiers in modal logic, Journal of Symbolic Logic 69 (2004), no. 3, pp. 879-906.

[13] Rosalie Iemhoff, On the admissible rules of intuitionistic propositional logic, Journal of Symbolic Logic 66 (2001), no. 1, pp. 281-294.

[14] - A(nother) characterization of intuitionistic propositional logic, Annals of Pure and Applied Logic 113 (2002), pp. 161-173.

[15] Intermediate logics and Visser's rules, Notre Dame Journal of Formal Logic 46 (2005), no. 1, pp. 65-81.

[16] Giorgi K. Japaridze, Modal logic means of investigation of provability, Ph.D. thesis, Lomonosov Moscow State University, 1986 (in Russian).

[17] Marcus Kracht, Book review of [20], Notre Dame Journal of Formal Logic 40 (1999), no. 4 , pp. $578-587$.

[18] Richard E. Ladner, The computational complexity of provability in systems of modal propositional logic, SIAM Journal on Computing 6 (1977), no. 3, pp. 467-480.

[19] Paul Lorenzen, Einführung in die operative Logik und Mathematik, Springer, 1955.

[20] Vladimir V. Rybakov, Admissibility of logical inference rules, Studies in Logic and the Foundations of Mathematics vol. 136, Elsevier, 1997.

[21] Construction of an explicit basis for rules admissible in modal system S4, Mathematical Logic Quarterly 47 (2001), no. 4, pp. 441-446.

[22] Edith Spaan, Complexity of modal logics, Ph.D. thesis, University of Amsterdam, 1993. 